\title{
Isolamento e caracterização dos componentes da hemoglobina de Mylossoma sp., um teleósteo da Amazônia(*)
}

\author{
Joseph P. Martin ('); Joseph Bonaventura ( $\left.{ }^{2}\right)$; Maurizio Brunori $\left({ }^{3}\right)$; Hans J. Fyhn ( $\left.{ }^{4}\right) ;$ Unni E. H. Fyhn (4);
} Robert L. Gaïlick $\left({ }^{5}\right)$; Dennis A. Powers $\left({ }^{6}\right)$; Michael T. Wilson (7)

\section{Resumo}

Foram isoladas duas hemoglobinas de um peixe caracídeo, Mylossoma sp. O componente eletroforético de maior migração anódica constitui $89 \%$ do hemolisado total. As duas hemoglobinas nativas têm peso molecular aparente de 57.000 por cromatografia em gel. Os pesos moleculares aparentes das subunidades desnaturadas são 14.000 por eletroforese em gel de dodecil sulfato de sódio. Não ocorre polimerização depois da oxidação com ferrocianeto de potássio. O estudo da união de oxigênio indica que o componente mais "anódico" da hemoglobina possui um efeito Root. Em pH 5,9 na presença de $1 \mathrm{mM}$ ATP a hemoglobina fica saturada apenas $45 \%$ quando equilibrada com ar a 1 atmosfera. O com. ponente mais anódico possui um efeito Bohr normal que é aumentado em presença de $1 \mathrm{mM}$ ATP. A cooperatividade, determinada por n na equação de Hill, varia com o pH. No $\mathrm{pH} 6,7$ e abaixo deste na presença de $1 \mathrm{mM}$ ATP, $\mathrm{n}<1$. A presença de $1 \mathrm{mM}$ ATP causa uma ređução em $\mathbf{n}$ em pH abaixo de 8,2. O comportamento menos anódico evidencia um comportamento muito diferente tendo um efeito Bohr reverso $\Delta \log \mathrm{P}_{1 / 2} / \Delta \mathrm{pH}=0,14$, entre $\mathrm{pH} 7,0$ e 8,0 o qual muda para um efeito Bohr normal, $\Delta \log \mathrm{P}_{1 / 2} / \Delta \mathrm{pH}=-0,13 \mathrm{com}$ adição de $1 \mathrm{mM}$ ATP. Esta hemoglobina mostra ccoperatividade em todos os valores de $\mathrm{pH}$ estudados. Não mostra efeito Root. Estudos de cinética rápida da ligação $\mathrm{CO}$ e da dissociação do $\mathrm{O}_{2}$ dos componentes isolados da hemoglobina mostraram que ambos processos são dependentes do pH para cada componente. Estes resultados são consistentes com as análises dos dados de equilíbrio do oxigênio. As hemoglobinas de Mylossoma sp. se assemelham às de Hoplosternum, truta, salmão, remora e cadozete, no grau de sua diferenciação funcional e podem representar especializações evolutivas designadas para servir funções fisiológicas diversas.

\section{INTRODUÇÃo}

As hemoglobinas de peixes têm sido intensivamente estudadas nos recentes anos. Elas são experimentalmente mais tratáveis que as hemoglobinas de mamífero em investigações cinéticas das transições conformacionais que acompanham as ligações e oferecem exemplos surpreendentes da adaptação molecular à fisiologia interna e ambiente físicos externos. A maioria dos sangues dos peixes contém várias hemoglobinas e foi sugerido que multiplicidade de proteínas possa ser fisiologicamente vantajosa (Riggs, 1970). Bonaventura et al. (1975) delimitam 3 categorias de hemoglobinas de peixes. A primeira classe inclui sistemas de hemoglobinas simples e múltiplas que são sensiveis à temperatura e ao $\mathrm{pH}$. Nos casos em que ocorrem hemoglobinas múltiplas, os componentes são relativamente não diferenciados com respeito a seu comportamento funcional. A segunda classe de hemoglobinas de peixes compreende sistemas de múltiplos componentes, cujas hemoglobinas individuais diferem marcadamente com respeito a propriedades funcionais. A terceira classe abrange hemoglobinas que são sensiveis ao $\mathrm{pH}$ mas não sensíveis à temperatura. Classe do sistema 2 foi cogitada para representar uma condição "avançada" na evolução das hemoglobinas dos peixes. Entretanto, dada a sua complexidade molecular, geralmente consistindo de 4 ou mais componentes, poucos sistemas como estes já foram descritos. Num esforço de amplificar as precedentes su-

(") - Versão original inglesa publicada em Comp. Blochem. Physiol. 62 A (1). 1979.

(1) - Department of Zoology, Duke University Marine Laboratory, Beaufort, North Carolina 28516.

(2) - Department of Biochemistry, Duke University Medi cal Center and Duke University Marine Laboratory, Beaufort, North Carolina 28516.

(3) - CNR Center for Molecular Biology, Institute of Chemistry and Biochemistry, Faculty of Medicine, University of - Rome, Rome, Italy.

(4) - Institute of Zoophysiology, University of Oslo, P.O. Box 1051, Blindern, Oslo, Norway.

(5) - Department of Zoology, University of Texas at Austin, Austin, Texas 78712 .

(6) - Department of Biology, Johns Hopkins University, Baltimore, Maryland 21218.

(7) - Department of Chemistry, University of Esses, NIvenhoe Park, Colchester CO4 3SQ, England. 
gestões, este trabalho sumariza as propriedades funcionais das hemoglobinas de Mylossoma sp., um peixe caracídeo que se encontra em rios tropicais, na América do Sul. O sistema de hemoglobinas de Mylossoma contém 2 componentes facilmente separáveis; cada qual se assemelha às hemoglobinas correspondentes de truta (Salmo irideus Brunori, 1975) e outra Classe II de hemoglobinas em certas facetas do seu comportamento cinético.

\section{MATERIAL E MÉTodo}

isolamento - Espécimes de Mylossoma sp. foram coletados em novembro e dezembro de 1976, durante uma expedição no R/V "Alpha Helix" no rio Amazonas, aproximadamente $50 \mathrm{~km}$ acima, no rio Solimões, da junção com o rio Negro. Os peixes foram colhidos em rede de arrasto e malhadeira e transportados para o "Alpha Helix" para esíudos. O sangue era obtido de punção cardíaca e colocado em seringas heparinizadas de vidro e geladas (100 $\mu \ell$ de heparina sódica, (5000 I.U./m $)$ em $1,7 \% \mathrm{NaCl}$ por $5 \mathrm{~m} \ell$ de sangue). Pequenas amostras foram tomadas em tubos capilares de $50 \mu \ell$ e colocadas por 3 minutos em uma centrífuga de soro para estimações dos hematócritos. As células vermelhas do sangue foram lavadas 3 vezes em 10 volumes de $1 \mathrm{mM}$ Tris gelado, $\mathrm{pH} 8,0,1,7 \% \mathrm{NaCl}$ e então lisadas em 3 volumes de $1 \mathrm{mM}$ Tris, $\mathrm{pH} 8,0$ por 1 hora a $\mathrm{O}^{\circ} \mathrm{C}$. Um décimo do volume de $1 \mathrm{M} \mathrm{NaCl}$ foi adicionado ao hemolisado e a mistura foi centrifugada a $28.000 \times \mathrm{g}$ por 15 minutos para remover restos celulares. O sobrenadante foi então retirado, sal e fosfatos orgânicos, por passagem através de uma coluna de Sephadex de $2,5 \times 50 \mathrm{~cm}$ resina G-25 equilibrada em 0,1 $\mathrm{mM}$ Tris, $\mathrm{pH} 8,5$ seguido de tratamento na coluna de deionização com as seguintes resinas de cima para baixo: $2 \mathrm{~cm}$ Dowex-50 W forma de amônio, $2 \mathrm{~cm}$ Dowex-1 forma acetato, $20 \mathrm{~cm}$ de um leito de resina misturada de Bio. Rad AG $501-X^{\prime}$ (D). A hemoglobina purificada foi mantida a $5^{\circ} \mathrm{C}$ até ser utilizada. Uma amostra de eritrócitos foi congelada a $-70^{\circ} \mathrm{C}$, transportada ef gelo seco para Beaufort, Carolina do Norte e mantida a $-20^{\circ} \mathrm{C}$ por dois meses. Em Beaufort, as células foram descongeladas, lisa- das e o peso molecular do derivado carbóxl da hemoglobina foi determinado por filtração em gel.

ABREVIAÇõEs - Bis-tris, bis (2 hidroxietil) imino tris (hidroximetil) metano; Tris, tris (hidroximetil) amino metano; ATP, adenosina trifosfato: EDTA, ácido etilenediaminetetraacetico; $1^{\prime}$, constante de velocidade de $2^{\mathrm{a}}$ ordem da combinação do monóxido de carbono; K. constante de $1^{\text {a }}$ ordem de velocidade de dissociação do oxigênio; $P_{1 / 2}$, pressão parcial do oxigênio na qual metade dos grupos heme aproveitáveis teve ligação de oxigênio.

PURIFICAÇÃo - $\mathrm{O}$ hemolisado fracionado (170 mg de proteína) foi trazido para $\mathrm{pH} 8,5$ em tampão 0,05 Tris- $\mathrm{HCl}$ e aplicado ao DEAE Sephadex A-50 coluna de troca iônica $(1,8 \times$ $20 \mathrm{~cm}$ ) equilibrada com o mesmo tampão. A separação ocorreu a $5^{\circ} \mathrm{C}$. A coluna foi lavada com equilibração de tampão e o $1 .^{\circ}$ pico eluiu imediatamente. A coluna foi então lavada com tampão $0,05 \mathrm{M}$ Tris-acetato $\mathrm{pH} \mathrm{6,2} \mathrm{e} \mathrm{o} \mathrm{segundo}$ pico de proteína foi obtido. Após a separação, o eluente contendo cada pico, foi concentrado por diálise contra $8 \%$ polietileno glicol M.W. 20.000 dissolvido em 1,0 mM Tris- $\mathrm{HCl}$ pH 8,0.

ELETROFORESE - Eletroforese vertical em $g \in I$ de poliacrilamida $(\mathrm{pH} 8,9,7,5 \%$ gels $)$ foi feita a temperatura ambiente de acordo com Davis (1964) e Ornstein (1964). Amostras de hemoglobina $(1 \mathrm{mg} / \mathrm{ml})$ em tampão superior contendo $0,1 \mathrm{M} \beta$-mercaptoetanol e uma pequena quantidade de ditionito foi borbulhado com monóxido de carbono e aplicado aos geis. Albumina sérica bovina foi usada como padrão de mobilidade. Os geis foram corados por 3 horas em Coomassie Brilliant Blue R" $25 \%$, ácido acético, metanol, solução aquosa $(1: 2: 4)$ e descorada por difusão. Os geis foram medidos a $560 \mathrm{~nm}$ usando um medidor de gel Gilford ligado a um monocromador Beckman DU.

ESTUDOS DO PESO MOLECULAR - EXperimentos de filtração, em gel foram feitos em hema lisados oxidados (tratados com $\mathrm{K}_{3} \mathrm{Fe}(\mathrm{CN})_{6}$ ) e em hemolisados oxigenados (reduzidos com (3-mercaptoetanol) numa coluna de Sepharose $4 B(2 \times 90 \mathrm{~cm})$ equilibrada com 0,05 M Tris, pH 7,5, 1 mM EDTA a $5^{\circ} \mathrm{C}$, de acordo com Fyhn e Sullivan (1975). Em adição o peso molecular 
do derivado carbóxi foi medido. Uma coluna de $1,6 \times 110 \mathrm{~cm}$ de resina ACA-34 (ProdutoresLKB) em 0,05 M Tris, pH 8,0, 1 mM EDTA a $5^{\circ} \mathrm{C}$ foi padronizada com catalase de fígado bovina, piruvato quinase, mioglobina do músculo radular de búzio (Busycon caniculatum), $\propto$-quimotripsinogênio - $A$ (pâncreas bovino), e mioglobina de cachalote. Os dados foram analisados pelo delineamento do volume de eluição de proteína vs. o log do peso molecular (Andrews, 1964). Amostras $(\approx 20 \mathrm{mg} / \mathrm{ml})$ foram aplicadas em $0,05 \mathrm{M}$ Tris, $\mathrm{pH} 8,0$ mais glicerol.

Pesos moleculares de cadeias de hemoglobina desnaturadas foram determinados por eletroforese em dodecil sulfato de sódio (SDS) de acordo com Weber e Osborn (1969) exceto que a solução de incubação foi em $6 \mathrm{M}$ uréia e $0,1 \mathrm{M}$ em $\beta$-mercaptoetanol. Transferina humana, albumina sérica bovina, ovalbumina, $\propto$-quimotripsinogênio-A, (pâncreas bovino), e mioglobina de cachalote foram usados como padrão de peso molecular. A albumina sérica bovina, catalase, $\propto$-quimotripsinogênio-A, ovalbumina, piruvato quinase e transferina foram obtidos da Companhia Química Sigma. A mioglobina de cachalote foi obtida da Companhia Miles-Serevac e a mioglobina do músculo radular foi presente do $\mathrm{Dr}$. Robert Terwilliger.

ESTUDOS DO EFETTO ROOT - Os componentes de hemoglobina foram examinados quanto à presença do efeito Root. As absorbâncias das soluções de hemoglobina tamponadas e equilibradas com ar $+1 \mathrm{mM}$ ATP foram monitoradas como uma função de $\mathrm{pH}$. Leituras das densidades ópticas em dois comprimentos de onda $(560,576 \mathrm{~nm})$ foram convertidas para saturação fracional pela seguinte equação e tirada a média.

\section{Aobservado- Adeoxi}

Amax oxi- Adeoxi

1. Adeoxi = absorbância da solução de hemoglobina desoxigenada no $\mathrm{pH}$ em estudo.

2. Amax oxi = absorbância obtida para solução de hemoglobina oxigenada a pH 8,6.

3. Aốbservado $=$ absorbância obtida para a solução de hemoglobine oxigenada no $\mathrm{pH}$ em estudo.
ESTUDOS DE EQUILÍBRIO DE OXIGÊNIO Equilíbrio do oxigênio em Mylossoma do sangue total foi efetuado a $30^{\circ} \mathrm{C}$ pelo método de Powers, et. al., (1978) usando um analisador de dissociação de oxigênio Hem-O-Scan (American Instrument $\mathrm{Co}$ ). $\mathrm{O}$ equilíbrio do oxigênio das hemoglobinas purificadas foi feito a $20^{\circ} \mathrm{C}$ como descrito em Riggs e Wolbach (1956). As soluçōes de hemoglobina (60 $\mu \mathrm{M}$ heme) foram levados para força iônica 0,05 em tampão Tris ou Bis-tris. $O$ valor de $\dot{P}_{1 / 2}$ foi determinado sob condiçōes de variação de $\mathrm{pH}$. Estes experimentos foram efetuados na presença e na ausência de $1 \mathrm{mM}$ ATP.

EXPERIMENTOS DE CINÉTICA RÁPIDA - EXperimentos de cinética foram efetuados com um aparelho Gibson-Durrum "stopped-flow" equipado com um passo de $2 \mathrm{~cm}$ de observação. Cinética de dissociação de oxigênio foi medida pelo método ditionito (Antonini \& Brunori, 1971) em presença e ausência de 1,25 mM ATP. A concentração heme nestes experimentos foi $10-20 \mu \mathrm{M}$ antes da mistura. A constante da velocidade de combinação para monóxido de carbono (CO) foi obtida pela mistura rápida de soluções desoxigenadas reduzidas de hemoglobina (10-20 $\mu \mathrm{M}$ em heme) em tampão Tris ou Bis-tris do phi desejado e força iônica com uma solução de concentração conhecida, $86 \mu \mathrm{M}$, de monóxido de carbono na água. Soluções de $\mathrm{CO}$ foram obtidas pela diluição quantitativa de $0,86 \mathrm{mM}$ de soluções estoque de água destilada equilibrada com gás a $1 \mathrm{~atm}$ e $30^{\circ} \pm 1^{\circ} \mathrm{C}$. Experimentos de ligação de $\mathrm{CO}$ foram efetuados como uma funçăo de pH com 1,25 mM ATP e sem ele, a reação foi estudada a uma concentração saturada de $\mathrm{CO}$ e analisada como um processo de pseudo $1^{\text {a }}$ ordem.

TAMPÕES - Todos os tampöes empregados em estudos de equilíbrio foram de força iônica 0,05 . Em experimentos de cinética, o tampão, após mistura, também era de 0,05 I. Tampōes Tris foram usados em experimentos com $\mathrm{pH}$ acima de 7,0 , enquanto tampões Bis-tris foram empregados em experimentos com $\mathrm{pH}$ abaixo de 7,0 . 


\section{Resultados}

CARACTERIZAÇÃO DOS COMPONENTES DE MYLOSSOMA - Com a eletroforese, a hemoglobina se mostrou em 2 bandas no gel de poliacrilamida (fig. 1). Dez indivíduos foram inspecionados e nenhuma variação neste padrão foi encontrada. Densitometria das células coradas revelou que o componente que mais migra para o polo positivo compreende $89 \%$ da hemoglobina total. Componente 1, a espécie de migração mais lenta, tem uma mobilidade relativa de 0,15 em comparação com a albumina sérica bovina, enquanto que a mobilidade relativa do componente 2 é 0,4 sob as mesmas condições. A média dos hematócritos de 8 espécimes foi $45 \pm 6 \%$.

A hemoglobina de Mylossoma elui como um único pico de peso molecular em experimentos de filtração em gel sob as condições

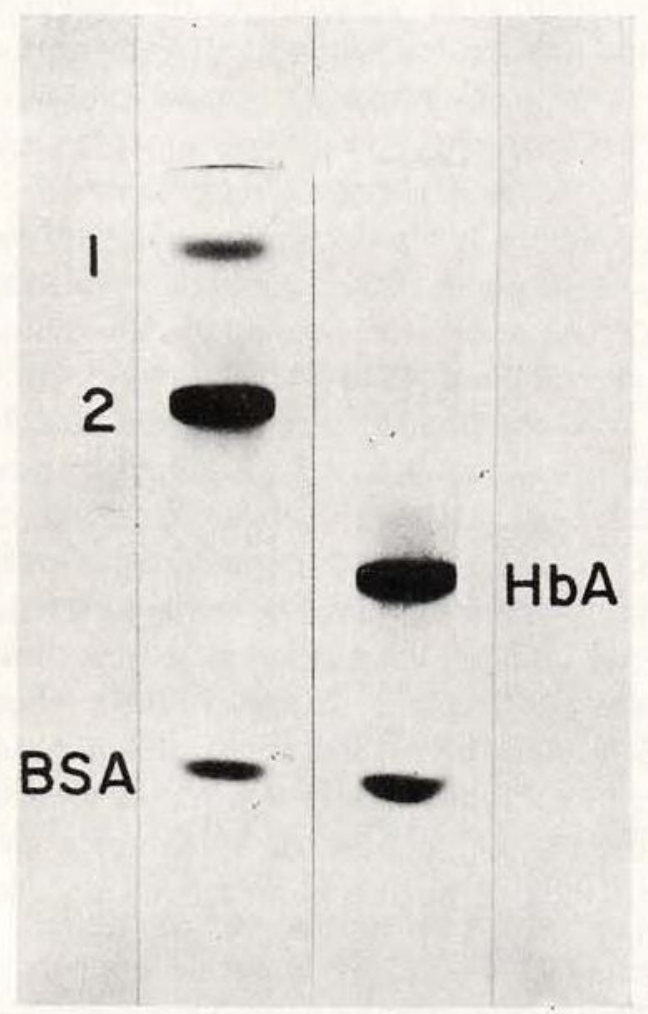

Fig. 1 - Discos de gel em poliacrilamida do hemolisado de Mylossoma e de hemoglobina humana. A esquerda, componentes 1 e 2 de hemoglobina de Mylossoma respectivamente são os mais próximos ao cátodo. A direita hemoglobina humana; o ânodo está localizado no final da figura. Albumina sérica bovina é a banda mais anódica em cada gel. de redução e oxidação. O peso molecular aparente da forma carboxi é 57.000 (Fig. 2). O peso molecular aparente das cadeias de hemoglobina desnaturadas foi 14.100 , determinado pela eletroforese de gel SDS. Este valor se compara favoravelmente ao peso molecular aparente da cadeia de hemorglobina humana de 14.600 obtido sob as mesmas condições.

A cromatografia em DEAE Sephadex separa o hemolisado do peixe em 2 zonas homogêneas simetricamente eluídas, picos 1 e 2 (fig. $3)$, correspondendo aos componentes 1 e 2 do disco eletroforético de gel respectivamente.

EQUILÍBRIO DE OXIGÊNIO - Uma parte da porcentagem de saturação do sangue de $M y$ lossoma a $30^{\circ} \mathrm{C}$ vs. o log da pressão parcial de oxigênio é apresentado na Fig. 4. A ligação de oxigênio é cooperativa e o efeito de Bohr é evidente. $A P_{1 / 2}$ do eritrócito é $12 \mathrm{~mm} \mathrm{Hg}$, a $\mathrm{pH} 7,6$. Desde que a hemoglobina não podia ser saturada com oxigênio sob condições de $5,6 \%$ de $\mathrm{CO}_{2}$ era impossível determinar a $\mathrm{P}_{1 / 2}$ sob estas condições. Entretanto, um decréscimo na afinidade da ligação de oxigênio da hemoglobina em presença de $5,6 \%$ de $\mathrm{CO}_{2}$ é notável.

A dependência do $\log \mathrm{P}_{1 / 2}$ e $n$ dos componentes 1 e 2 fracionadas da hemoglobina em $\mathrm{pH}$ e concentração de ATP a $20^{\circ} \mathrm{C}$ é ilustrada na Fig. 5, $\mathrm{A}$ e B. Estimativas do $\mathrm{P}_{1 / 2}$ e $n$ foram calculadas pelos quadrados mínimos de regressão dos dados de equilíbrio para a equação de Hill. O componente 2 mostra um efeito de $\mathrm{pH}$ e de fosfato. $\mathrm{O} \mathrm{P}_{1 / 2}$ na hemoglobina fracionada aumenta aproximadamente 80 vezes entre $\mathrm{pH} 8,3$ e 5,9 , mudando de $0,63 \mathrm{~mm} \mathrm{Hg}$ para $50,2 \mathrm{~mm} \mathrm{Hg}$. Existe uma considerável dispersão nos dados de equilíbrio em hemoglobina separadas tornando impossível uma acurada determinação da magnitude do efeito Bohr. O efeito Bohr medido em presença de $1 \mathrm{mM}$ ATP entre $\mathrm{pH} 7,0$ e 8,0 é aproximadamente $\Delta$ Log $\mathrm{P}_{1 / 2} / \Delta \mathrm{pH} \equiv 0,9$. O efeito do ATP é desprezível em $\mathrm{pH}$ alto e gradualmente aumenta em potência com o abaixamento do $\mathrm{pH}$, aumentando o efeito Root em valores de $\mathrm{pH}$ abaixo de 7,0 . A mudança na $P_{1 / 2}$ a pH 7,6 causada pela adição de $1 \mathrm{mM}$ ATP para o componente 2 fracionado, é de $0,80 \mathrm{~mm} \mathrm{Hg}$ para $1,4 \mathrm{~mm} \mathrm{Hg}$. Uma alteração de 214 vezes no $P_{1 / 2}$ ocorre entre $\mathrm{pH} 8,6$ e 5,9 em presença de $1 \mathrm{mM}$ ATP. 


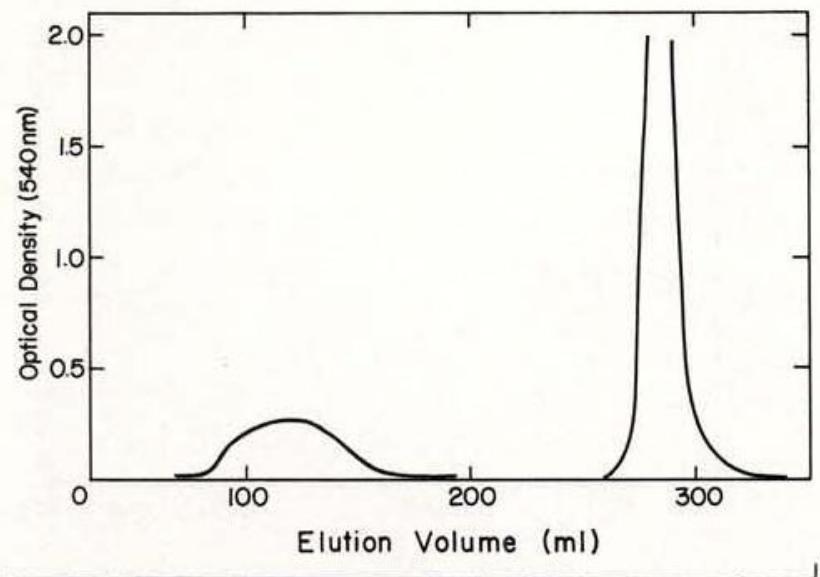

Fig. 2 - Cromatografia em DEAE Sephadex do hemolisado de Mylossoma. As condições estão descritas no texto.

O grau de cooperatividade também varia com o $\mathrm{pH}$. Um valor $n$ de 1,0 é obtido para o componente 2 fracionado a $\mathrm{pH} 5,9 ; n$ aumenta para 2,0 a $\mathrm{pH} 7,3$ e monotonicamente declina para 1,2 a pH 9,1. Adições de ATP abaixam o valor de $n$ sob $\mathrm{pH} 8,2$; a pH $7,6 \mathrm{n}=1,4$, a $\mathrm{pH}$ $5.9 n$ cai para 0,5 .
$O P_{1 / 2}$ do componente 1 fracionado aumenta com aumento de $\mathrm{pH}$. O efeito Bohr, entre pH 7,0 e 8,0 , é $\Delta \log \mathrm{P}_{1 / 2} / \Delta \mathrm{pH}=+0,14$. O ATP reverte completamente o efeito Bohr re-

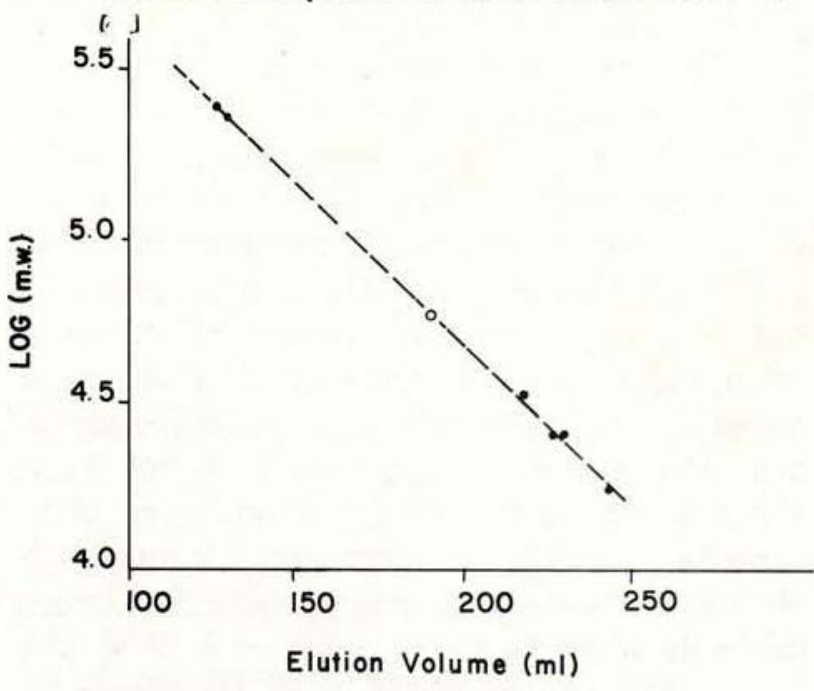

Fig. 3 - Curva padrão de proteína para cromatografia de gel ACA-34 da carbóxi hemoglobina de Mylossoma. (O) Hemoglobina de Mylossoma. Os padrões estão descritos no texto, em ordem decrescente de peso molecular.

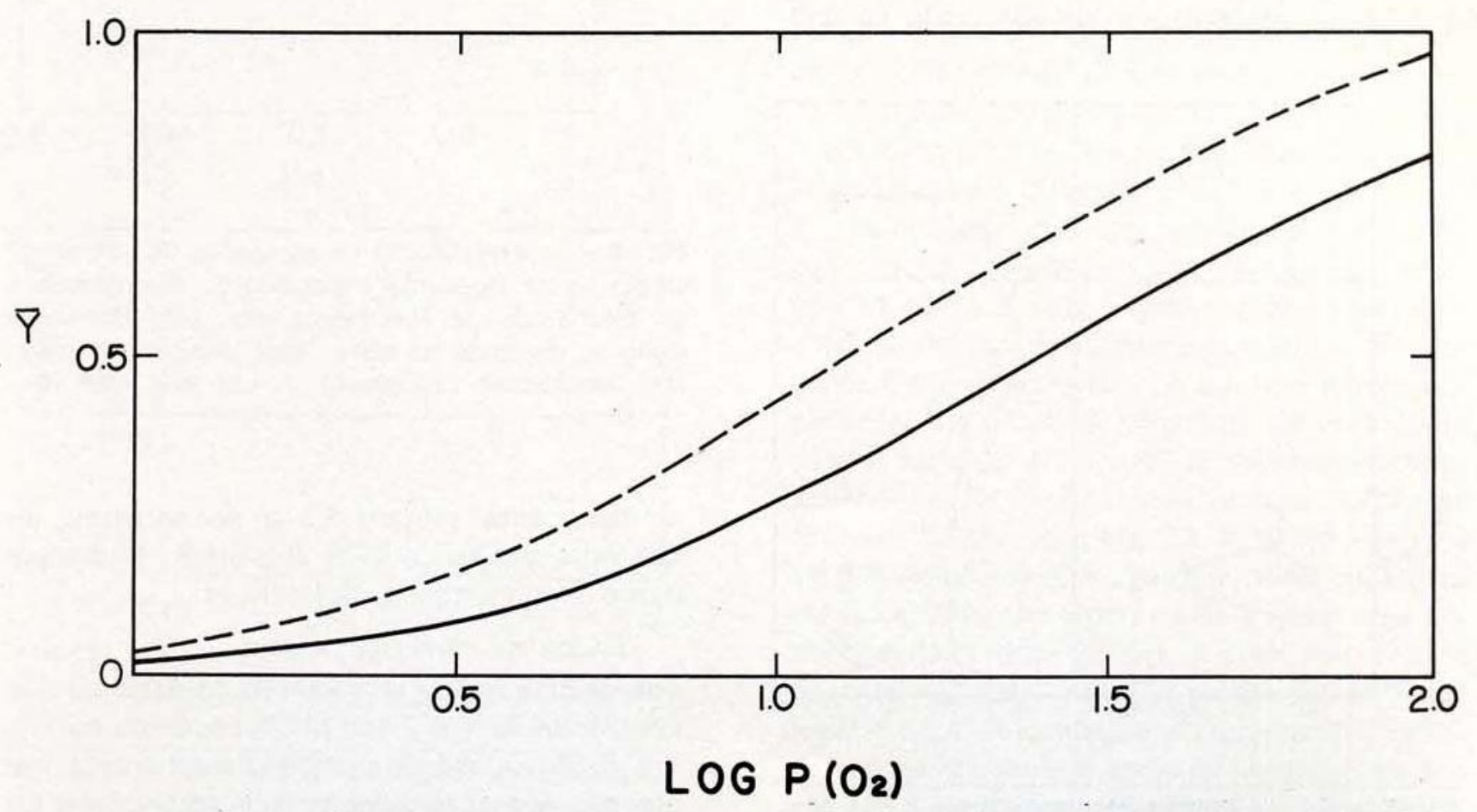

Fig. 4 - Curvas de oxigenação do sangue total de Mylossoma a pH 7,6 e pH 7,0 a $30^{\circ} \mathrm{C}$. Curva 1 a pH 7,6 (-..) e curv̄a 2 a pH 7,0 (-) representa a contínua oxigenação em ausência e presença de $5,6 \%$ de $\mathrm{CO}^{2}$ respectivamente. A curva medida a $5,6 \%$ de $\mathrm{CO}^{2}$ não se aproxima à assyntota superior à pressão parcial do oxigênio atmosférico. Deste modo, sua posição exata é incerta. 
verso tal que $\Delta \log \mathrm{P}_{1 / 2} / \Delta \mathrm{pH}$ se torna aproximadamente $-0,1$ a $-0,15$ entre $\mathrm{pH} 6,7$ e 8,15 . A pH 9,1, nenhum efeito significativo de ATP é aparente, mas a $\mathrm{pH} 6,7$ o $\mathrm{P}_{1} / 2$ muda de 1,3 $\mathrm{mm} \mathrm{Hg}$ para $4,1 \mathrm{~mm} \mathrm{Hg}$, um aumento de 3-vezes. O componente 1 é cooperativo em todos os valores de $\mathrm{pH}$ examinados em ambas as soluções de hemoglobina fracionada e solução de hemoglobina fracionada $+1 \mathrm{mM}$ ATP. O efeito do ATP é aumentar a cooperatividade na maioria dos valores de $\mathrm{pH}$. O componente 1 fracionado liga oxigênio menos rapidamente em $\mathrm{pH}$ altos que o componente 2. Contudo, a $\mathrm{pH}$ baixo, esta afinidade é consideravelmente mais alta que no componente 2: $\mathrm{A} \mathrm{pH} \mathrm{9,1} \mathrm{a}$ afinidade da hemoglobina fracionada do componente 1 é $37 \%$ do componente 2, mas sob $\mathrm{pH} 7,0$, a afinidade do componente 1 é maior que a do componente 2 .

Desde que ATP aumenta o efeito de Root no componente 2, a porcentagem de oxigenação de soluções de hemoglobina equilibrado com ar foi medido como uma função de $\mathrm{pH}$ a $20^{\circ} \mathrm{C}$ em presença de $1 \mathrm{mM}$ ATP (Fig. 6). Tensões atmosféricas de oxigênio são insuficientes para saturar todos os sítios heme sob $\mathrm{pH} 7,5$. A percentagem de saturação cai pre-

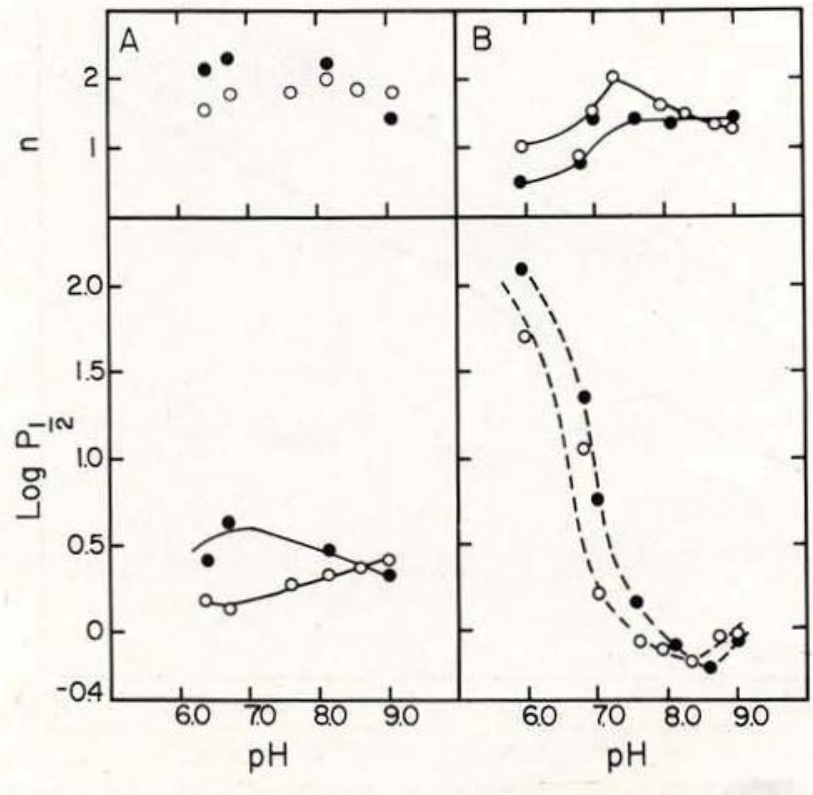

Fig. 5, a \& b - A dependência do $P_{1} / 2$ e n dos componentes', $1,5 \mathrm{~A}$ e $2,5 \mathrm{~B}$, a $\mathrm{pH}$ a $20^{\circ} \mathrm{C}$. Hemolisado fracionado $(\mathrm{O})$ e hemolisado fracionado $+1 \mathrm{mM}$ ATP (•) de Mylossoma. Condições estão descritas no texto.

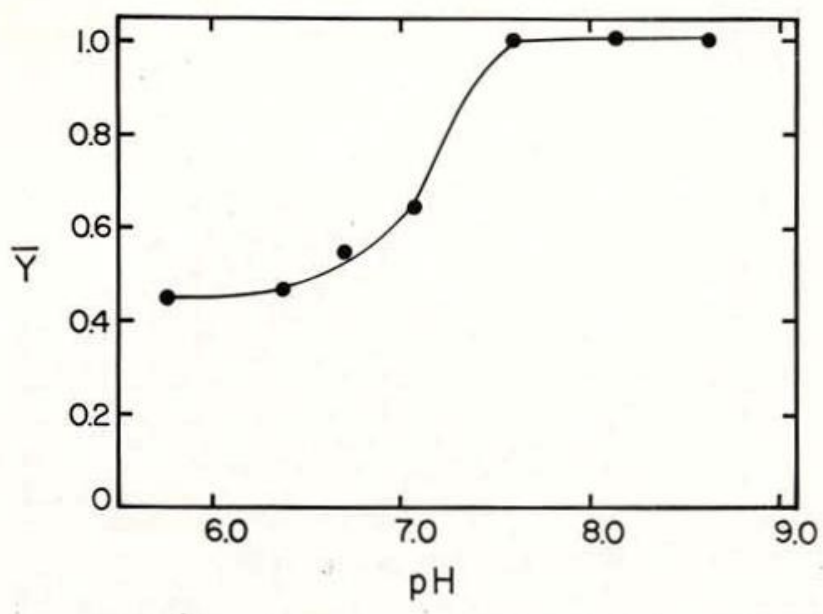

Fig. 6-A saturação fracionada $Y$, das soluções de hemoglobina não fracionada equilibradas com ar como uma função de pH em presença de 1 mM ATP.

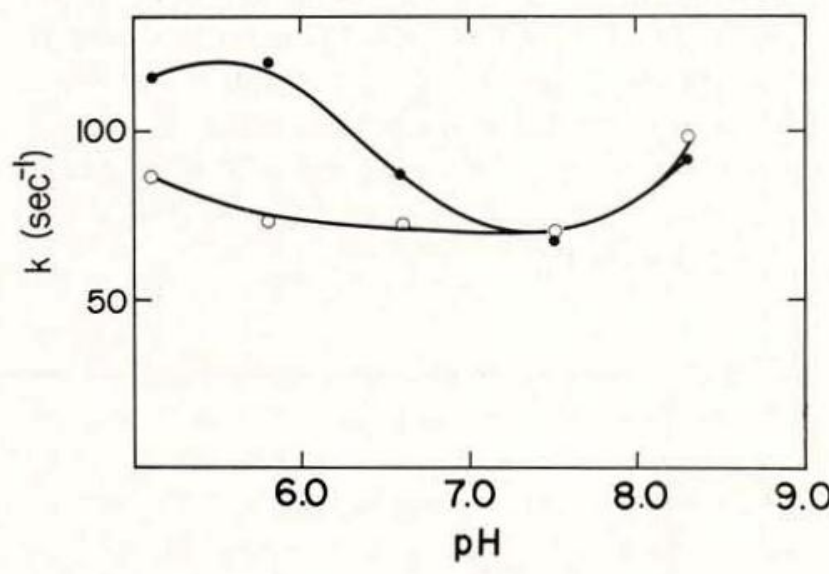

Fig. 7-A dependência da constante (K) de dissociação de 1." ordem para oxigênio do componente 1 de hemolisado de Mylossoma com pH. Condições como as descritas no texto. Hemolisado fracionado (O), hemolisado fracionado $+1,25 \mathrm{mM}$ ATP (•).

cipitadamente sob pH 7,5 e se aproxima de um valor abaixo de $45 \%$ sob $\mathrm{pH} 6$. O componente 1 não amostra efeito Root.

DADOS DE CINÉTICA RÁPIDA - Os resultados da cinética de dissociação do oxigênio dos componentes 1 e 2 sob pH 8,0 aparece na Fig. 7 e 8 . A velocidade para o componente 1 fracionado é aproximadamente insensível ao $\mathrm{pH}$ desta ordem. Entretanto, a pH 8,3 a velocidade inicial aumenta para $98 \mathrm{seg}^{-1}$ e a reação tornase autocatalítica. Sob pH 8,0 a reação é homogênea. Adição de ATP não altera o padrão do 
comportamento autocatalítico mas a velocidade de dissociação aumenta em baixo $\mathrm{pH}$, de $69 \mathrm{seg}^{-1}$ a pH 7,5 para $120 \mathrm{seg}^{-1}$ a pH 6,0. A reação aparece fora do nível a ou abaixo $\mathrm{pH} 6$. $\mathrm{O}$ componente 2 exibe um marcado efeito de $\mathrm{pH}$, aumentando a velocidade de $17 \mathrm{seg}^{-1}$ para

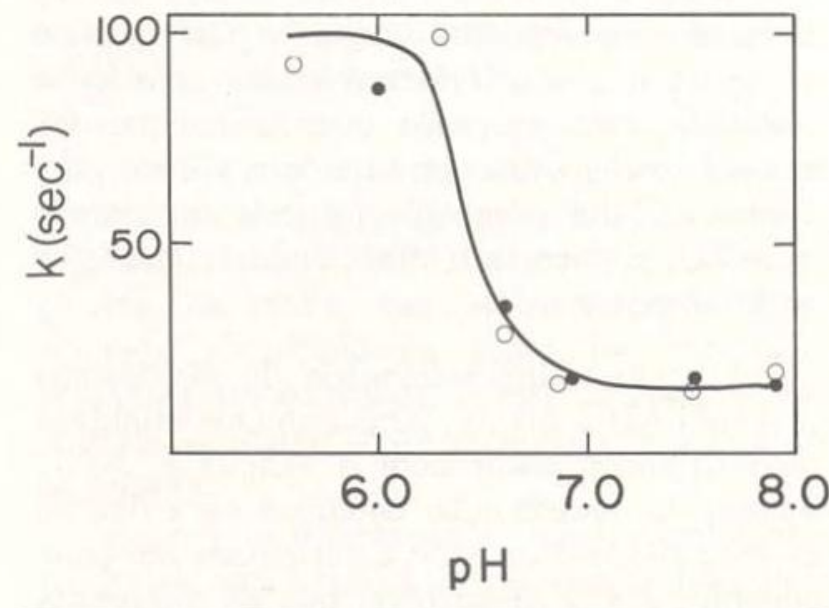

Fig. 8-A dependência da constante (K) de dissociação de $1 .^{a}$ ordem para oxigênio do componente? da hemoglobina de Mylossoma. Condiçōes como as descritas no texto. Hemolisado fracionado (O), hemolisado fracionado $+1,25 \mathrm{mM}$ ATP ()

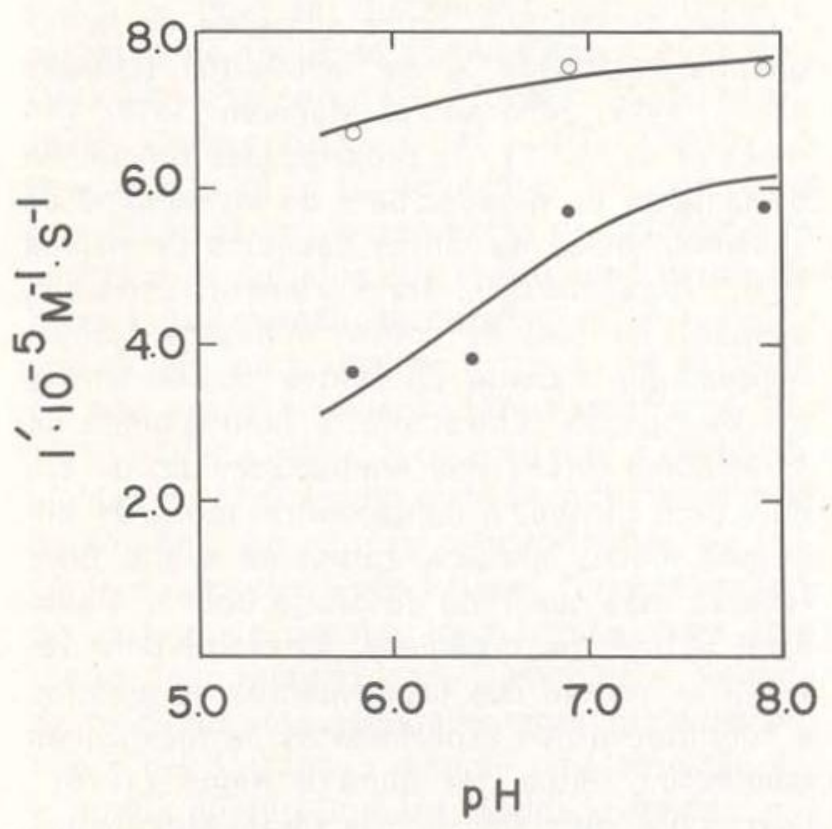

Fig. 9-A constante de velocidade de combinação de 2.a ordem ( $\left.l^{\prime}\right)$ do monóxido de carbono do componente 1 da hemoglbina de Mylossoma vs. pH. Condięões como as descritas no texto. Hemolisado fracionado $(\mathrm{O})$ e hemolisado fracionado $+1,25 \mathrm{mH}$ ATP (•).

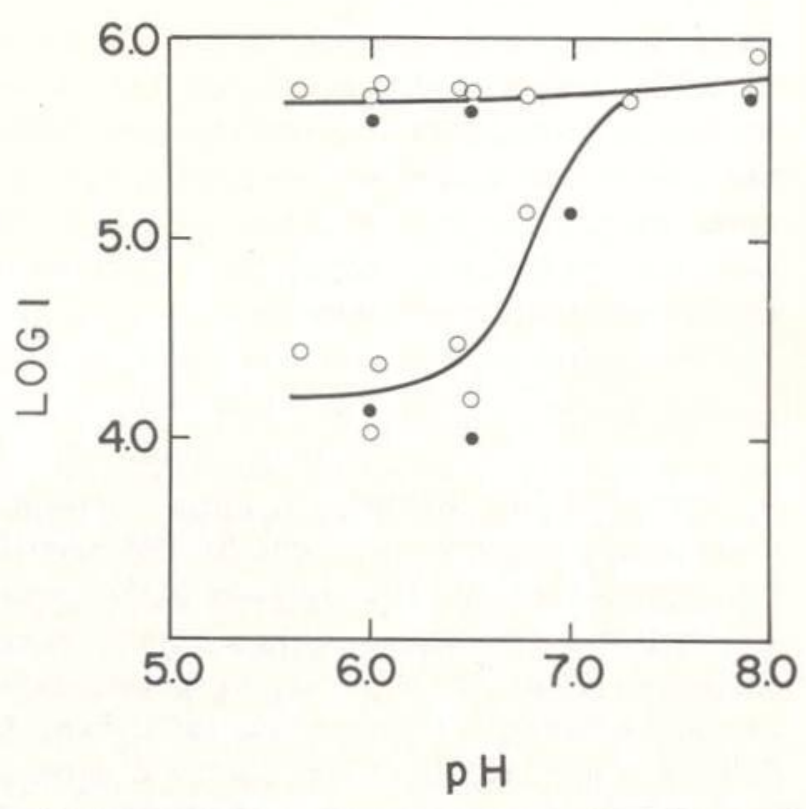

Fig. 10 - O logarítmo da constante de velocidade de dissociação de $2 .^{a}$ ordem (1') do monóxido de carbono do componente 2 da hemoglobina de Mylossoma vs. pH. Condiçōes como as descritas no texto. Hemolisado fracionado $(\mathrm{O})$, hemolisado fracionado $+1,25 \mathrm{mM}$ ATP (๑)

$99 \mathrm{seg}^{-1}$ entre $\mathrm{pH} 7,0$ e 6,3. Acima de $\mathrm{pH} 7,0$ e abaixo de $\mathrm{pH} 6,0$, há um pequeno efeito de $\mathrm{pH}$ na velocidade. ATP não mostra nenhum efeito na velocidade de dissociação do oxigênio sob $0 \mathrm{pH}$ estudado. A reação de dissociação foi homogênea em qualquer $\mathrm{pH}$.

Velocidades de combinação de monóxido de carbono a vários valores de $\mathrm{pH}$ para componentes 1 e 2 estão apresentadas na Fig. 9 e 10. A reação de combinação para o componente 1 não é autocatalítico e é homogêneo em $\mathrm{pH}$ entre 5,8 e 8,0 . A constante de velocidade para o material fracionado é relativamente insensível a $\mathrm{pH}$ aumentando apenas $6,7 \times 10^{5}$ $\mathrm{M}^{-1}$ seg $^{-1}$ a pH 5,8 para $7,5 \times 10^{5} \mathrm{M}^{-1} \mathrm{seg}^{-1}$ a $\mathrm{pH}$ 8,0. Adição de ATP abaixa a média constante de velocidade entre estes $\mathrm{pHs}$ e induz uma notável sensibilidade ao $\mathrm{pH}$. A taxa aumenta de $3,6 \times 10^{5} \mathrm{M}^{-1} \mathrm{seg}^{-1}$ a pH 5,8 e $5,8 \times 10^{5} \mathrm{M}^{-1} \mathrm{seg}^{-1}$ a $\mathrm{pH} 8,0$. A velocidade de combinação de $\mathrm{CO}$ para o componente 2 torna-se heterogênea sob $\mathrm{pH} 7,0$. Acima deste $\mathrm{pH}$ a reação é homogênea. As velocidades de ambas as fases rápida e lenta sob pH 7 são plotadas. A fase rápida é insensivel ao $\mathrm{pH}$ e ao ATP. A fase lenta é sensível ao pH entre 6,5 a 7,5 . A pH 6,0 a veloci- 
dade da fase rápida é aproximadamente de 32vezes da fase lenta. Entretanto, nenhum efeito de ATP na velocidade é visto. As duas fases são provavelmente devidas ao desacoplamento entre as cadeias $\propto$ e $\beta$ apesar das amplitudes das duas fases, enquanto comparáveis, não serem do mesmo tamanho.

\section{DISCUSSÃo}

Nenhuma variabilidade genética, determinada pela eletroforese de gel foi descoberta nos componentes da hemoglobina Mylossoma. Polimorfismo genético é um fenômeno comum em hemoglobinas de espécies de peixes temperados e boreais (Frydenborg, 1965; Fyhn \& Sullivan, 1974; Hjorth, 1975; Hiorth \& Simonsen, 1975; Bonaventura et al., 1976) mas é comparativamente raro nas espécies de peixes amazônicos (Fyhn et al., 1978). Os pesos moleculares da hemoglobina de Mylossoma nativa e desnaturada se assemelha àqueles obtidos para outras hemoglobinas de teleósteos, assim como o padrão de eluição dos hemolisados tratados com $\mathrm{K}_{3} \mathrm{~F}_{\mathrm{e}}(\mathrm{CN})_{6}$ e $\beta$-mercaptoetanol em cromatografia de gel. Os hematócritos de sangue de Mylossoma caem na ordem superior dos valores relatados para outras espécies amazônicas (Johansen \& Magnum, 1978) .

A afinidade de oxigênio do sangue total a $\mathrm{pH} 7,6,30^{\circ} \mathrm{C}$ é, algumas vezes, mais baixa que as encontradas em outras espécies amazônicas (Johansen \& Magnum, 1978; Powers et al., 1978) ainda mais altas que afinidades de sangue e eritrócitos relatados para muitas espécies norte americanas (e.g., truta de rio, Black et. al., 1976; "rainbow" truta, Cameron, 1971; cavala, Prosser, 1973; salmão, Prosser, 1973: "wahoo", tuna "bigeye", albacora, tubarão, 1975). A água do rio Solimões é mais quente, mais hipóxica e hipercárbica do que a dos meio ambiente marinho ou dos rios norte americanos mais rápidos (Johansen \& Mangnum, 1978; Fisher, 1978). Deste modo, as espécies de peixes que habitam o rio Solimões, pode esperar-se, possuem hemoglobinas com mais altas afinidades pelo oxigênio que as dos peixes que vivem em águas mais geladas, ambientes melhor oxigenados (Krogh \& Leitch, 1919; Hochachka \& Somero, 1971; Johansen \& Magnum, 1978). Entretanto, o gênero Mylos- soma consiste de espécies ativas, de águas médias. Tais espécies foram relatadas como possuindo hemoglobinas que mostram grandes efeitos de $\mathrm{pH}$ e relativa pouca afinidade ao oxigênio (Hashimoto et al., 1960; Yamaguchi et al., 1962, 1973; Black, 1966; Riggs, 1970; Cameron 1971; Brunori et al., 1974). As observações precedentes podem explicar porque os eritrócitos de Mylossoma têm uma baixa afinidade pelo oxigênio quando comparadas aơs de outras espécies amazônicas, mas relativamente alta afinidade quando comparada àquelas, previamente mencionadas, espécies norte americanas.

A hemoglobina fracionada do Mylossoma $+1 \mathrm{mM}$ ATP a pH $7,6,20^{\circ} \mathrm{C}$ tem uma afinidade pelo oxigênio maior com o sangue a $30^{\circ} \mathrm{C}$. Apesar da contribuição entálpica para ligação de oxigênio não ter sido determinada em componentes 1 e 2, é possível que as diferenças da temperatura experimental contribuam em parte para as diferenças de afinidade observadas. Também moduladores como inositol pentafosfato e guanosina trifosfato podem estar presentes em eritrócitos e mais adiante ampliem as diferenças em afinidades de oxigênio entre soluções de hemoglobina fracionada e os eritrócitos (Isaacks et al., 1977; Johansen \& Magnum, 1978; Torracca et al., 1977). As propriedades funcionais do sistema de hemoglobina de Mylossoma se assemelham às de outras espécies de peixes (i.e., Hoplosternum, truta, remora, cadozete, salmão) no fato de conter múltiplos componentes, que exibem diferentes comportamentos de ligação. Entretanto, a hemoglobina de Mylossoma difere das enumeradas acima em que, pelo menos, o componente que é de migração menos anódica apresenta efeito Bohr reverso mas que falta de efeito Bohr e é sensível a fosfatos orgânicos. O efeito Bohr reverso se reflete nos experimentos de cinética e equilíbrio e nos experimentos de focalização isoelétrica feitos por Bunn \& Riggs (1978). Outras espécies amazônicas foram simultaneamente descobertas possuindo hemoglobinas com efeitos Bohr reverso (i. e. Pteryplichthys sp., Brunori et al., 1978; Hoplosternum littorale, Garlick et al., 1978); e o efeito Bohr reverso tinha sido previamente relatado de hemoglobinas de enguias (Gillen \& Riggs, 1973; Weber 
et al., 1975). O efeito está caracteristicamente associado a hemoglobinas de anfíbios, tendo sido notificada por Urodeles, Amphiuma means, Bonaventura, et al., (1977), Triton cristatus, Morpurgo, et al., (1970); "Caecilians", Typhlonectes compressicauda, Garlick et al., (1978) e em Ranidae, girinos de Rana cafesbeiana, Watt e Riggs (1975). Nestes sistemas, pequenos anions e fosfatos orgânicos geralmente anulam ou mesmo revertem o efeito Bohr reverso. Por outro lado, a hemoglobina catódica de truta é insensível a fosfatos organicos. Brunori et al. (1975) mostraram na hemoglobina de truta que esta insensibilidade aumenta alcançando-se como um resultado impedimentos estéricos, à falta de complementaridade de carga entre o modulador e o sítio de ligação.

E um fato interessante que hemoglobinas de peixes com efeito Bohr reverso tenham sido encontrado em peixes amazônicos. Conforme afirmação anterior, muitas destas espécies atravessam períodos de "stress" de oxigênio. A presença de hemoglobinas fosfato-sensitivas associada com a regulação fisiológica da concentração de fosfato intracelular tem sido evocada como um mecanismo para auxiliar a extração de oxigênio de ambientes hipóxicos (Wood \& Johansen, 1972, 1973a, b; Wood et al., 1975; Bonaventura et al. 1977; Greany \& Powers, 1978). Similarmente, hemoglobinas com efeito Bohr reverso estão associadas com espécies de anfíbios que vivem em habitats de baixa concentração de oxigênio e se acredita que ajudem ao animal na extraçäo de oxigênio de ambientes hipóxicos (Bonaventura et. al., 1977). Deste modo, o componente 1 da hemoglobina de Mylossoma pode permitir a retirada do oxigênio quando as concentraçōes de oxigênic do ambiente são baixas. Complementando, o fato de possuir uma hemoglobina com efeito Bohr reverso pode aumentar a retirada de oxigênio pelo sangue durante períodos em que o pH do sangue diminui uma situaçâo encontrada durante intensa atividade física.

A maior contribuição cinética, para o efeito Bohr reverso em $\mathrm{pH}$ acima de 7,5 no componente 1 da hemoglobina fracionada, encontra-së na velocidade de dissociação do oxigênio a qual diminui quando o $\mathrm{pH}$ diminui. Entretanto, os dados não explicam o decréscimo ulte- rior no $\mathrm{P}_{1} / 2$ baixo $\mathrm{pH} 7,5$ assim como a velocidade de dissociação de oxigênio, nem a alteração da velocidade de combinação de $\mathrm{CO}$ satisfatoriamente a $\mathrm{pH}$ de ordem inferior. Talvez a ligação de $\mathrm{CO}$ não imite completamente o processo de ligação de oxigênio na hemoglobina de Mylossoma. A indução de um efeito Bohr normal por $1 \mathrm{mM}$ ATP se reflete em ambas as velocidades de $\mathrm{CO}_{\text {on }}$ e $\mathrm{O}_{2 \text { off. }}$.

O hemolisado de todos os membros da família Characidae, examinados por Farmer, et al. (1972), exibem efeitos Root. Como apontados pelos autores, o papel fisiológico do efeito Root, nestes peixes, não é o bombeamento de gás para dentro da bexiga natatória, desde que os peixes que possuem bexigas natatórias não possuem a "rete mirabile". 0 único aparelho de bombeamento de gás que se encontra neles é o da rede coróide do olho. A afinidade do componente 2 muda para acima de 200-vezes entre $\mathrm{pH} 6,3$ e 8,6 . A sensibilidade ao $\mathrm{pH}$ do equilíbrio do oxigênio se reflete tanto na velocidade de combinaçỏes como na constante de dissociação do oxígênio. Entretanto, nem a constante cinética é sensível ao ATP, em marcado constraste com os resultados de equilíbrio. Em outras espécies (i.e., "spot", truta, carpa) o ATP geralmente afeta a constante de dissociação do oxigênio, com um efeito máximo ocorrendo a $\mathrm{pH}$ baixo. A velocidade de dissociação de $\mathrm{O}_{2}$ da hemoglobina de "spot" excede 300 seg $^{-1}$ a pH baixo como determinado pela espectofotometria "stoppedflow" (Bonaventura et al., 1976); a hemoglobina com o efeito Root da truta atinge uma velocidade de dissociação de $\mathrm{O}_{2}$ de $1100 \mathrm{seg}^{-1}$ a pH 7,0 como determinado pelos estudos de variação de temperaturas (Giardina et al., 1973). O valor máximo medido do componente 2 de Mylossoma foi $99 \mathrm{seg}^{-1}$. As duas fases da dissociação do oxigênio provavelmente resultam das diferenças inerentes às propriedades de ligação das cadeias $\propto$ e $\beta$. O desempareIhamento cinético das cadeias tipo $\propto$ e $\beta$ da hemoglobina de Mylossoma se manifesta na heterogeneidade da velocidade de combinação do monóxido de carbono a pH abaixo de 7,0 e em valores baixos de $n$ observados em experimentos de equilíbrio a $\mathrm{pH} 5,9$. E possível que a dissociação de oxigênio da hemoglobina de Mylossoma seja heterogênea a pH baixos con- 
tendo uma fase lenta não sensível e fosfatos orgânicos e fase rápida dependente de ATP. a qual é perdida no aparelho "stopped-flow". Tal heterogeneidade foi relatada para a hemoglobina de um prosímio Lemur fulvus fulvus (Bonaventura et al., 1974) e estaria mais de acordo com os nossos dados de equilíbrio, mas, no momento, não podemos oferecer nenhuma evidência para isto.

Ulteriores evidências para a semelhança do sistema de hemoglobina Mylossoma com o da truta surgiram dos experimentos imunológicos de Reichlin \& Davis (1978). O componente 1 de Mylossoma reage fortemente em reação cruzada no teste de dupla difusão com anti-soro preparado com hemoglobina catódica de truta. Nenhuma reação cruzada é observada entre o componente 1 e o anti-soro específico para a hemoglobina $\mathrm{pH}$-sensível da truta. A situação contrária foi obtida para o componente 2 de Mylossoma; naquela a reaçäo cruzada reage fortemente com anti-carpa mas não com soro anti-truta I. Poder-se-ia pensar que as semelhanças na função da hemoglobina podem ter sido acompanhadas por semelhanças na estrutura da proteína imunologicamente detectáveis e evolutivamente conservadas.

\section{CONCLUSÃo}

O sistema de hemoglobina de Mylossoma se assemelha aos de espécies de peixes ativas, as quais vivem no hemisfério setentrional em seu padrão de composição molecular e comportamento funcional. Deste modo, múltiplos sistemas de hemoglobina, designados para preencher uma série de demandas metabólicas, pode ter evoluído como uma solução geral para o problema da complexidade metabólica em peixe. Entretanto, a relativamente alta afinidade com oxigênio da hemoglobina de Mylossoma e a presença de um componente com efeito Bohr reverso parece ser caracteristico de certas espécies amazônicas e pode auxiliar estes peixes na obtenção de oxigênio de um ambiente hipóxico.

\section{AGRADECIMENTOS}

Maurižlo Brunori expressa seus agradecimentos ao Conselho Nacional de Pesquisa (C. N. R.) da Itália pelo apoio financeiro.
Joseph Bonaventura reconhece agradecidamente as concessões do Instituto Nacional de Saúde dos Estados Unidos e da Fundação Nacional de Ciência. Michael $T$. Wilson reconhece agradecidamente as viagens permitidas pela "Royal Society" e a "Duke Biomedical RSG Grant" do Instituto Nacional de Saúde. Unni E.H. Fyhn reconhece uma concessão de viagem do Conselho Norueguês de Pesuisa para Ciência e Humanidades. Gostaríamos de agradecer ao governo brasileiro por permitir-nos fazer pesquisa em suas águas. Joseph Bonaventura reconhece agradecidamente o apoio do "ONR Contract ND-0014-75-C-0190". Apoio adicional foi dado por "NIH grant BM-21314" (para A. Riggs) .

\section{SUMMARY}

The two hemoglobins of a characid fish, Mylossoma sp. have been isolated. The more anodally migrating electrophoretic component constitutes $89 \%$ of the total hemolysate. The two native hemoglobins have apparent molecular weights of 57,000 by gel chromatography. The apparent molecular weights of the denatured subunits are 14,100 by sodium dodecyl sulfate gel electrophoresis. No polymerization occurs after oxidation with potassium ferricyanide. Oxygen binding studies indicate that the more anodal hemoglobin component possesses a Root effect. At pH 5.9 in the presence of $1 \mathrm{mM}$ ATP the hemoglobin is only $45 \%$ saturated when equilibrated with air at one atmosphere. The more anodal component possesses a normal Bohr effect which is enhanced in the presence of $1 \mathrm{mM}$ ATP. The cooperativity, as determined by $n$ of the Hill equation varies with $\mathrm{pH}$. At and below $\mathrm{pH}$ 6.7 in the presence of $1 \mathrm{mM}$ ATP, $\mathrm{n}<1$. The presence of $1 \mathrm{mM}$ ATP causes a reduction in $\mathrm{n}$ below $\mathrm{pH}$ 8.2. The less anodal component evinces very different behavior having a reverse Bohr effect $\Delta \log \mathrm{p}_{1 / 2} \Delta / \mathrm{pH}=0.14$, between $\mathrm{pH} 7.0$ and 8.0 which changes to a normal Bohr effect, $\Delta \log p_{1 / 2} / \Delta$ $\mathrm{pH}=-0.13$ upon addition of $1 \mathrm{mM}$ ATP. This hemoglobin shows cooperativity, at all $\mathrm{pH}$ values studied. It does not show a Root effect. Rapid kinetic studies of $\mathrm{CO}$ binding and $\mathrm{O}_{2}$ dissociation of the isolated hemoglobin components show that both processes are $\mathrm{pH}$ dependent for each of the components. These results are consistent with the analysis of the oxygen equilibrium data. Mylossoma hemoglobins resemble those of Hoplosternum, trout, salmon, sucker and loach in the degree of their functional differentiation and may represent evolutionary specializations designed to serve diverse physiological functions. 


\section{BIBLIOGRAFIA}

ANDREWS, $\mathbf{P}$.

1974 - Estimation of the molecular weights of proteins by Sephadex gel filtration. Biochem. J., 91:222-232.

ANTONINI, E. \& BRUNori. M.

1971 - Hemoglobin and Myoglobin in Their Reactions with Ligands. North Holland, Amsterdam. $436 \mathrm{p}$.

BLACK, E.C.; KIRKPATRIK, D. \& TUCKer, H.H.

1966 - Oxygen dissociation curves of the blood of brook trout (Salvilinus fontinalis) acclimated to summer and winter temperatures. J. Fish. Res. Brd. Canad., 23:1-13.

Bonaventura, C.; Sullivan, B. \& Bonaventura, J. 1974 - The effects of $\mathrm{pH}$ and anions on functional properties of hemoglobin from Lemur fulvus fulvus. J. Biol. Chem., 249:3768-3775.

Bonaventura, C.; Sullivan, B.; Bonaventura, J. \& BOURNE, S.

1977 - Anion modulation of the negative Bohr effect of hemoglobin from a primitive amphibian. Nature, 265:474-476.

Bonaventura, C.; Sullivan, B.; Bonaventura, J. \& BRUNORI, M.

1976 - Spot hemoglobin, studies on the root effect hemoglobin of a marine teleost. J. Biol. Chem., 251:1871-1876.

Bonaventura, J.; Bonaventura, C. \& Sullivan, B.

1975 - Hemoglobins and hemocyanins. Comparative aspects of structure and function. J. Exp. Zool., 194:155-174.

BRUNORI, $\mathbf{M}$.

1975 - Molecular adaptation to physiological requirements: the hemoglobin system of trout. Curr. Topics in Cell Regulation, 9:1-39.

BRUNoRi, M.; BONAVENTURA, J.; BONAVENTURA, C.; GIARDINA, B.; BOSSA, F. \& ANTONIN1, E.

1974 - Hemoglobins from trout: structural and functional properties. Molec and Cell. Biochem., 1:189-196.

BRUNori, M.; BONAVENTURA, J.; Focesi JR.; A.; GALDAMES-PORTUS, M.I. \& WILSON, M.T.

1978 - Separação e caracterizaçāo dos compo nentes de hemoglobina de Pterygoplichthys pardalis, o acaribodó. Acta Ama zonica. $8(4)$ : Suplemento. (Este volume).

Brunori, M.; Falcioni, G.; Fortuna, G. \& Giardina, B. 1975 - The effects of anions on the oxygen binding properties of the hemoglobin components from trout. (Salmo irideus) Arch. Biochem. Biophys, 168:512-519.
BUNN, H.F. \& RigGs, A.

1978 - A medida do efeito Bohr em hemoglobinas de peixe por focalização elétrica em gel. Acta Amazonica 8(4): Suplemento. (Este volume).

CAMERON, J.W.

1971 - Oxygen dissociation characteristics of the blood of the rainbow trout, Salmo gairdneri. Comp. Biochem. Physiol, 38A: 699-704.

Duvis, B.J.

1964 - Disc electrophoresis - II. Method and application to human serum proteins. Ann. N. Y. Acad. Sci., 121:404-427.

FARMER, M.; FYhN, H.J.; FYhN, U.E.H. \& NOBLE, R.W. 1978 - Ocorrência de hemoglobinas de eferto Root em peixes amazônicos. Acta Ama zonica $8(4)$ : Suplemento. (Este volume)

FISHER, T.R.

1978 - Plâncton e produção primária em sistemas aquáticos da bacia da Amazônia Central. Acta Amazonica 8(4) : Suplemento. (Este volume).

Frydenboro, O.

1965 - The hemoglobin polymorphism in Norwegian cod populations. Hereditas, 53:257-271.

Fyhn, U.E.H. \& Sullivan, B.

1974 - Hemoglobin polymorphism in fishes. I. Complex phenotypic patterns in the toadfish Opsanus tau. Biochemical Genetics, 11:373-385.

FYhn, U.E.H. \& Sullivan, B.

1975 - Elasmobranch hemoglobins: Dimeriza tion and polymerization in various species. Comp. Biochem. Physiol. 50B; 119-129.

FYHN, U.E.H.; FYHN, H.J.; DAVIS, B.J.; POWERS, D.A.; FINK, W.L. \& GARLICK, R.L.

1978 - Heterogeneidade de hemoglobina nos peixes da Amazônia. Acta Amazonica 8(4) : Suplemento. (Este volume).

GARLICX, R.L.; BUNN, H.F.; FYHN, H.J.; FYHN, U.E.H.; MARTIN, J.P.; Noble, R.W. \& POWers, D.A.

1978 - Estudos funcionais dos componentes separados da hemoglobina de um bagre de respiração aérea, Hoplosternum littorale. (Hancock). Acta Amazonica 8(4): Suplemento. (Este volume).

GARLICK, R.L.; DAVIS, B.J.. FARMER, M.; FYhN, H.J.; FYhN, U.E.H.; NOBLE, R.W.; POWERS, D.A.; RIGGS, A. \& WEBER, R.E.

1978 - Uma troca materno-fetal no equilíbrio de oxigênio das hemoglobinas dos caecilios vivíparos, Typholonectes compressisicauda. Acta Amazonica 8(4):Suplemento. (Este volume) 
Giardina, B.; Brunori, M.; Binotti, J.; Glovenco, S. \& ANTONINI, $\mathbf{E}$.

1973 - Studies on the properties of fish hemoglobins; kinetics of reaction with oxygen and carbon monoxide of the isolated hemoglobin components from trout (Salmo irideus) Eur. J. Biochem., 39: 571-579.

GILLEN, R. \& RIGGS, A.

1973 - Structure and function of the isolated hemoglobin of the American eel, Anguilla rostrata. J. Biol. Chem., 248 : 1961-1969.

Greaney, G.S. \& Powers, D.A.

1978 - Allosteric modifiers of fish hemoglobıns. In vitro and in vivo studies of the effect of ambient oxygen and $\mathrm{pH}$ on erythrocyte ATP concentrations. J. Exp. Zool. (In Press).

Hashimoto, K. Yamaguchi, Y. \& MatsuUra, F.

1960 - Comparative studies on two hemoglobins of salmon - IV. Oxygen dissociation curves. Bull. Jap. Soc. Fish, 26:827-834.

HJORTH, J.P.

1975 - Molecular and genetic structure of multiple hemoglobins of the eelpout, Zoarces viviparus L. Biochemical Genetics, 13:379-391.

HJORTH, J. \& SIMONSEN, V.

1975 - Genetics of Zoarces populations VIII. Geographic variation common to the polymorphic loci HBI and EST III. Hereditas, 81:173-184.

HochachKa, P. \& SOMERo, G.N.

1971 - Biochemical adaptation to the environment. In: Fish Physiology, 4, pp. 99-156.

Isacks, R.E.; KIM, H.D.; BArtlett, G.R. \& HARKNESS, D.R.

1977 - Inositol pentaphosphate in erythrocytes of a freshwater fish, pirarucu (Arapaima gigas). Life Sciences, 20:988-990.

Johansen, K. \& Mangum, C.P.

1977 - Respiratory properties of the blood of Amazon fishes. J. Exp. Zool. (In press)

KROGH, A. \& LEITCH, I.

1919 - The respiratory function of blood in fishes. J. Physiol., 52:288-300.

Morpurgo, G.; Battaglia, P.A. \& Leggio, T.

1970 - Negative Bohr effect in newt hemolysates and its regulation. Nature, 225:76-77.

ORNSTEIN, $\mathrm{I}_{\mathrm{k}}$

1964 - Disc electrophoresis - I. Background and theory. Ann. N. Y. Acad. Sci., 121: 321-349.
POWERS, D.A.

1974 - Structure, function and molecular ecology of fish hemoglobins. Ann. N. Y. Acad. Sci., 241:472-490.

Powers, D.A.; FyHn, H.J.; Fyhn, U.E.H.; MARTIN, J.P. Garlick, R.L. \& WoOd, S.C.

1978 - Estudo comparativo de equilíbrio de oxigênio no sangue de 40 gêneros de peixes da Amazônia. Acta Amazonica 8(4): Suplemento. (Este volume).

Presser, C.L.

1973 - Comparative Animal Physiology. 3rd ed. Philadelphia, W. B. Saunders Comp. $966 \mathrm{p}$.

REICHLIN, M. \& DAVIS, B.J.

1978 - Relações antigênicas entre peixes comuns à bacia do rio Amazonas. Acta Amazonica 8(4): Suplemento. (Este volume) .

RIGGs, A.

1970 - Properties of fish hemoglobins. In: Fish Physiology, v. 4:209-251.

Riggs, A. \& WOLBach, R.A.

1956 - Sulfhydryl groups and the structure of hemoglobin. J. Gen. Physiol., 39:585-605.

SHARP, G.D.

1975 - A comparison of the $\mathrm{O}_{2}$ dissociation properties of some scombrid hemoglobins. Comp. Biochem. Physiol., 51(A): 683-693.

Torracca?, A.M.; RAschetti, R.; Salviola, R.; RICIOIARDI, G. \& Winterhalter, K.H.

1977 - Modulation of the root effect in goldfish by ATP and GTP, Biochem. Biophys. Acta. 496:367-373.

WATT, K.W.K. \& RIGGS, A.

1975 - Hemoglobins of the tadpole of the bullfrog, Rana catesbeiana. Structure and function of the isolated components. J. Biol. Chem., 250:5934-5944

WeBER, K. \& OsBORN, M.

1969 - The reliability of molecular weight determinations by dodecyl sulfatepolyacrylamide electrophoresis. J. Biol. Chem., 244:4406-4412.

Weber, R.E.; LyKKeboe, G. \& Johansen, K.

1975 - Physiological properties of eel hemo globin: hypoxic acclimation, phosphate effects and multiplicity. J. Exp. Biol., $64: 475-488$.

WOOD, S.C. \& JOHANSEN, K.

1972 - Adaptation to hypoxia by increased HB $\mathrm{O}_{2}$ affinity and decreased red cell ATP concentration. Nature New Biologia, 237:278-279. 
WOOD, S.C. \& JOHANSEN, K.

1973a - Organic phosphate metabolism in nucleated red cells influence of hypoxia on eel $\mathrm{HB} \mathrm{O}_{2}$ affinity. Neth. J. Sea. Res., 7:328-338.

WOOD, S.C. \& JOHANSEN, K.

1973b - Blood oxygen transport and acid-base balance in cells during hypoxia. Am. J. Physiol., 225:849-851.

WOOD, S.C.; JohANSEN, K. \& Weber, R,

1975 - Effect of ambient $\mathrm{PO}_{2}$ on $\mathrm{Hh} \mathrm{O}_{2}$ affinity and red cell ATP concentrations in a benthic fish Pleuronectes platissa. Resp. Physiol., 25:259-267.

Yamaguchi, H.; YAmaguchi, K. \& MatsuURA, F.

1963 - Studies on two hemoglobins of loach II. Oxygen dissociation curve. Bull. Japan. Soc. Sci. Fisheries, 29:180-188.

YАMAGUCH, K.; КосніYамА, Y.; НАSHIMOTо, К. \& MATSUURA, F.

1962 - Studies on the multiple hemoglobins of eel II. Oxygen dissociation curve and the relative amounts of components F\&S. Bull, Japan. Soc. Sci. Fisheries, 28:192-200. 